\title{
Gestión del conocimiento organizacional: un encuentro necesario entre plataformas digitales, comunicación, educación y cultura*
}

\author{
Doris Elena Muñoz Zapata**, Juan Esteban Valencia Rey***
}

\begin{abstract}
Resumen
Para alcanzar una adecuada gestión de sus conocimientos, muchas organizaciones se concentran en implementar plataformas digitales que permitan el intercambio de información, dejando por fuera el componente humano. Este artículo de reflexión se ocupa de plantear una visión complementaria, a partir de la comunicación interna como activadora de interacciones grupales, empoderamiento y valores propicios en la cultura organizacional para el logro de los distintos niveles requeridos al gestionar conocimiento. Las conclusiones se concentran en las potencialidades de la estrategia comunicacional para fortalecer la cultura de la organización y a los individuoscolaboradores, facilitándoles exteriorizar, compartir y generar conocimiento organizacional.
\end{abstract}

Palabras clave: gestión del conocimiento, TIC, educación, cultura, comunicación.

\section{Knowledge Management in Organizations: a Necessary Encounter of Digital Platforms, Communications, Education and Culture}

\begin{abstract}
Aiming to achieve an adequate knowledge management many organizations focus on implementing digital platforms that allow information exchanges, but they leave the human component away. In this reflection paper a complementary vision is proposed, based on internal communication as the booster of group interactions, empowering and proper
\end{abstract}

values within the organizational culture for achieving the levels required when knowledge is managed. The conclusions are concentrated on the potentialities of the communication strategy to strengthen the organizational culture and also strengthen the individuals-employees, making the exteriorization, sharing and generation of organizational knowledge.

Key words: knowledge management, IT, education, culture, communication.

Gestão do conhecimento organizacional: um encontro necessário entre plataformas digitais, comunicação, educação e cultura

\section{Resumo}

Para atingir uma adequada gestão de seus conhecimentos, muitas organizações se concentram em implementar plataformas digitais que permitam o intercâmbio de informação, deixando por fora o componente humano. Este artigo de reflexão se ocupa de propor uma visão complementar, a partir da comunicação interna como ativadora de interações grupais, empoderamento e valores propícios na cultura organizacional para o lucro dos diferentes níveis requeridos ao gerir conhecimento. As conclusões se concentram nas potencialidades da estratégia comunicacional para fortalecer a cultura da organização e aos indivíduos-colaboradores, facilitando-Ihes exteriorizar, compartilhar e gerar conhecimento organizacional.

Palavras chave: gestão do conhecimento, TIC, educação, cultura, comunicação.

* Artículo derivado del proyecto en el cual se analizaron los modelos de gestión del conocimiento de 20 grandes empresas (más de 200 empleados, de acuerdo con la Ley 590 de 2000) del Valle de Aburrá.

** Magíster en Comunicación y Educación, docente investigadora, Facultad de Comunicación Social-Periodismo, Universidad Pontificia Bolivariana.doris.munoz@upb.edu.co.

*** Comunicador Social- Periodista, pasante de Investigación. Maestría en Comunicación Organizacional, Universidad Pontificia Bolivariana, juanesrey33@gmail.com. 


\section{Introducción}

El conocimiento, como pilar para el desarrollo de las sociedades, revierte cada vez mayor interés para los diversos actores de las mismas; al respecto, las organizaciones del sector productivo incorporan a sus contextos la necesidad imperante de transformar la información que circula a diario por sus redes y canales en aprendizajes con sentido, que enriquezca a sus recursos humanos, para una incidencia notoria en términos de mejoras e innovaciones en sus procesos, que puedan impactar de manera favorable, en su posicionamiento en los mercados y en sus capacidades para adaptarse y crecer en entornos caracterizados por la incertidumbre y el exceso de competencia.

A partir de las indagaciones realizadas por el proyecto de investigación: "roles de la comunicación organizacional en la gestión del conocimiento en empresas del Valle de Aburrá" se encontró que, pese a las empresas hacen inversiones significativas en la adquisición y montaje de plataformas digitales que sirvan de soporte al intercambio, almacenamiento y conversión de la información en conocimiento, se enfrentan a grandes dificultades en cuanto a la disposición de sus empleados al uso y apropiación de esos recursos, que en muchas ocasiones se asocian a carga laboral adicional y terminan subutilizados sin lograr sus propósitos centrales.

Esa visión limitada de la gestión del conocimiento que se concentra principalmente en la adquisición de tecnología para la circulación de la información coincide con el planteamiento de Arboníes:

La miopía hará que muchas empresas clausuren rápidamente el proyecto de gestión del conocimiento con un programa de software, centrando todo el esfuerzo en la gestión eficiente de la información, persiguiendo el sueño de la automatización de operaciones, incluida la conectividad y el intercambio de conocimientos (Arboníes, 2007, 7).

En la búsqueda de caminos complementarios para el logro de los propósitos en materia de gestión del conocimiento muchas organizaciones recurren a la realización de capacitaciones en el manejo de las plataformas dispuesta para tal fin, con la expectativa de que la dificultad pueda radicar en el desconocimiento de dichas tecnologías y sus funciones; incluso, en algunas empresas se han ideado estímulos para el personal, que puede recibir bonificaciones adicionales por participar activamente en estos procesos; sin embargo, las respuestas por parte de los recursos humanos siguen estando distantes de las proyectadas.

Los procesos de experimentación que viven las organizaciones al intentar gestionar su conocimiento centrándose en los componentes tecnológicos y digitales motivan la reflexión frente a la necesidad de reenfocar las prioridades en dichos procesos, dando una mirada prioritaria a las personas que hacen parte de los mismos, aunque las pistas de actuación sean limitadas en ese sentido:

Es de uso común formular la idea de que en el conocimiento lo más importante son las personas, aunque luego el éxito que se tiene en construir propuestas desde esta afirmación es más bien escaso porque se manejan relaciones informaciónpersona desde una óptica de procesos...el conocimiento no se podrá entonces gestionar en el sentido clásico de la palabra, sino que lo que se podrá gestionar será el proceso en que se intercambia y crea conocimiento (Arboníes, 2006, 68).

Dado el contexto anterior, es indispensable la reflexión y análisis frente a aspectos comportamentales, culturales y comunicacionales que pueden incidir favorablemente en la motivación de las personas involucradas en los procesos requeridos para la exteriorización, intercambio y generación de nuevos conocimientos en las organizaciones.

\section{Propuestas para modelos de gestión del conocimiento centrados en lo humano}

En el diseño e implementación de modelos de gestión del conocimiento, es indispensable la valoración de aspectos sociales y culturales de la propia organización que propende por la generación de esquemas ajustados a sus realidades y características particulares. 
A partir de las indagaciones realizadas en el proceso investigativo, se reconoce la enorme cantidad de modelos teóricos que la gestión del conocimiento posee (Pérez-Montoro, 2008), entre los que se destaca una mirada centrada en el proceso administrativo (Valhondo, 2010), tal como en el modelo Europeo CEN, el Cuadro de mando integral (Balance Scorecard) y el modelo de Demerest, así como su versión modificada por McAdam y McCreedy, pero no hay un desarrollo profundo y adecuado sobre la incidencia del factor humano y el desarrollo social (Valhondo, 2010). Por eso, se recomienda un desarrollo estructurado para el aspecto social de la gestión del conocimiento que incluya las actitudes del colaborador frente al conocimiento, los procesos de comunicación que alimentan las interacciones sociales en las que se forma el conocimiento y la cultura organizacional.

Según Nonaka y Takeuchi:

[...] el conocimiento es creado solo por los individuos. Una compañía no puede crear conocimiento sin individuos. La empresa apoya a individuos creativos o provee los contextos que necesitan para que creen conocimiento. Por tanto, la creación de conocimiento organizacional debe ser entendida como un proceso que amplifica organizacionalmente el conocimiento creado por los individuos (Nonaka \& Takeuchi, 1999, 65).

Se ha de tener en cuenta que las personas que interactúan en las organizaciones no necesariamente son conscientes del conocimiento que poseen, ya que generalmente es conocimiento personal, de contexto específico, subjetivo, experiencial y altamente práctico, llamado conocimiento tácito (Nonaka \& Takeuchi, 1999). Esto hace que sea difícil de comunicar y formalizar. Por otro lado, está el conocimiento que las organizaciones promueven, que es objetivo, sistemático, en lenguaje formal, racional y sistemático, que se conoce como explícito. Estos tipos de conocimiento fluyen en los mensajes y en el intercambio de información cotidianos de los equipos de trabajo. Dependiendo de la interacción, estos conocimientos pueden pasar de un tipo a otro, es decir, dejar de ser conocimiento tácito para ser conocimiento explícito, o viceversa. Según Nonaka y Takeuchi (1999), esta interacción puede darse de cuatro formas: socialización, exteriorización, interiorización y combinación.

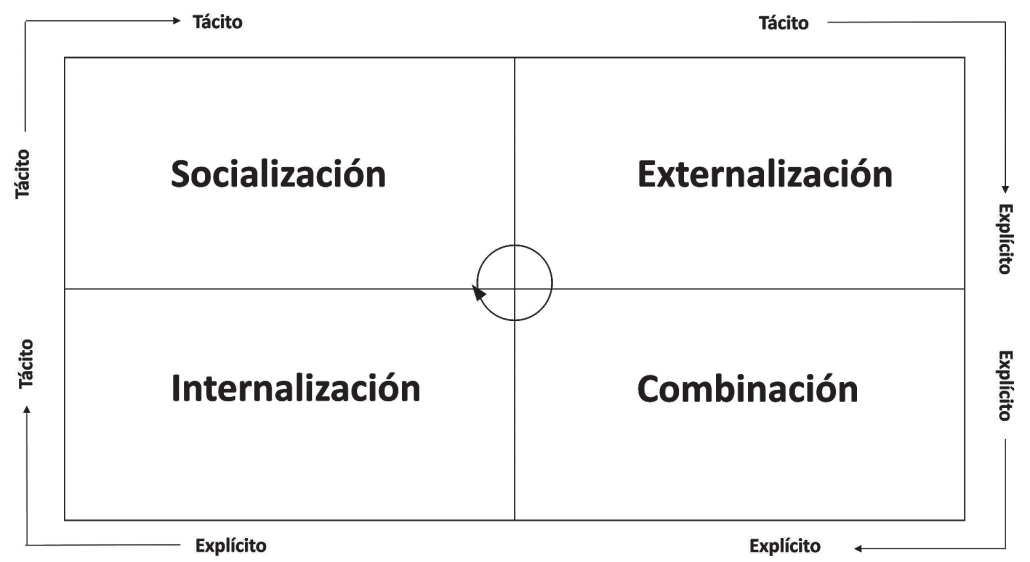

Figura 1. Cuatro formas de conversión del conocimiento

Fuente: Nonaka \& Takeuchi $(1999,69)$.

Estas formas de conversión están traspasadas por un componente comunicativo que permite la interacción social en la que se da la transformación del conocimiento. En el proceso de socialización que se compone de experiencias, pasa el conocimiento tácito de un individuo a otro, en espacios de contextualización en los que se comunican, generalmente de 
manera no verbal, códigos culturales sobre ser, sentir y actuar. La exteriorización o externalización pasa conocimiento tácito a explícito a través de enunciar ese conocimiento en conceptos, hipótesis, modelos, analogías o metáforas, es decir, dándole sentido y lógica a ese conocimiento, y lo hace a través de una comunicación fluida, bidireccional y simétrica en espacios de diálogo y reflexión grupal. Por otro lado, la combinación sistematiza conceptos usualmente organizados en un sistema de conocimiento y los mezcla con otros sistemas de conocimiento hasta que se armonicen. Para ello, es necesaria la formación de los individuos, el diálogo fluido sobre el conocimiento, así como nuevas formas de capacitación y formación en un clima de confianza para compartir lo que se sabe. Este proceso también puede darse a través de la educación. Por último, la interiorización, que podría compararse con la experiencia o el know how, es la apropiación del conocimiento explícito a través de la acción o puesta en uso del conocimiento hasta volverlo conocimiento tácito. Esto requiere un proceso interpretativo a través de lo comunicativo que le da sentido al conocimiento explícito en el quehacer diario.

Estas formas de conversión del conocimiento están intrínsecamente conectadas, a través del individuo, al grupo social y, más adelante, a la organización y viceversa, es decir, la interacción social. La gestión del conocimiento se da en la medida en que se pongan a rotar esas formas de conversión del conocimiento como una espiral a través de esa interacción.

\section{Actitudes de los empleados para la gestión del conocimiento}

Es necesario moldear y reforzar las actitudes básicas del individuo-colaborador hacia el conocimiento; actitudes como la apertura, la disposición a la participación, el empowerment y el aprendizaje continuo hacen parte del perfil clave que debe identificarse en la arquitectura del conocimiento (Awad \& Ghaziri, 2004).

Estas actitudes soportan y apoyan la gestión del conocimiento. Por tanto, se necesita una estrategia organizacional consistente y clara, capacidad comunicativa para compartirla y habilidades para incentivar estas actitudes en el empleado (Preciado, 2007). Sin embargo, esto solo es posible si se apoya en la cultura organizacional y la comunicación, ya que depende de las facilidades que el colaborador descubre en el ambiente para expresar esas actitudes. Al ser la participación un compromiso, un contrato voluntario con los demás miembros del grupo, se contrae también una responsabilidad en los resultados que lleva consigo más carga laboral en términos operativos y emocionales (Quintanilla Pardo, 2003). Pero también se asume un papel en la toma de decisiones que regala cierta autonomía en el día a día que se puede apoyar en el empowerment, porque "persigue que el personal tome las decisiones que afectan sus propios trabajos, sin necesidad de consultarlas con los jefes, o de esperar que ellos las tomen" (Preciado, 2007, 37). Eso exige a las organizaciones, principalmente a los jefes y directivos, establecer escenarios de participación y empoderamiento en donde se desarrollen esas habilidades.

Estas habilidades fortalecen la capacidad del empleado de elegir acciones en las que haya aprendizaje continuo, así como la identificación de necesidades de capacitación que garanticen los procesos de conversión del conocimiento en sí mismo y su equipo de trabajo. Pero, a la vez, esos procesos de conversión de conocimiento garantizan una participación continua, un adecuado empoderamiento y capacidad de asombro ante el conocimiento porque apoyan decisiones más asertivas según la estrategia organizacional, el contexto y las necesidades percibidas del área o proceso.

El aprendizaje continuo debe enfocarse, sin excluir los otros procesos, en la organización y el entorno organizacional, desde dos tipos de conversión que sirven para la contextualización: la socialización y la externalización. Parte de ese conocimiento radica en comprender las potencialidades y limitaciones de las estructuras de la organización. Para ello, son fundamentales los procesos de inducción tanto generales como del área. Por último, ese aprendizaje continuo radica en adaptar los conocimientos técnicos y profesionales que el empleado tiene a los contextos organizacionales, de tal manera que aporten significativamente para la generación 
de conocimiento y una toma de decisiones de acuerdo con ello.

La apertura al conocimiento, tanto para su creación como para su gestión, está anclada en el sentido de pertenencia y compromiso con los ideales de la organización en la dinámica cotidiana de los equipos de trabajo. Eso exige que se dé el proceso espiral con la estrategia organizacional, de tal manera que el empleado la socialice, externalice, combine e interiorice. Con ello asegura un "marco de referencia acerca de lo que es, hace y persigue la organización, que permite actuar con autonomía" (Preciado, 2007,38 ), a la vez que le ayudará a determinar qué es conocimiento valioso para los equipos de trabajo y la organización, así como para sí mismo en el contexto organizacional.

\section{Una cultura organizacional flexible y abierta al conocimiento}

\section{Según López, Marulanda e Isaza:}

La cultura organizacional es fundamental para la gestión del conocimiento en cualquier tipo de organización, que se entiende generalmente como el conjunto de valores, principios, ideas, costumbres, hábitos $\mathrm{y}$ tradiciones que son compartidos por todas las personas que hacen parte de una organización (López, Marulanda \& Isaza, 2011, 130).

Todo esto configura el contexto donde la persona gestionará el conocimiento. Esta cultura basa su existencia en la historia vivida desde su nacimiento y en las personas que han liderado y trabajado en ella, así como los paradigmas y criterios para la construcción de los procesos organizacionales que han formado la estructura. Su rol radica, según Schein (1988), en buscar la adaptación con el entorno y la integración en el interior de la organización.

Capriotti (2009) la define como lo real, lo que la organización es, lo que condiciona y caracteriza lo que la organización quiere ser. Según el autor, cimentado en Schein, las creencias, los valores y las pautas de conducta son los que dan orden a la cultura porque está compuesta por ellos. No obstante, la conformación de una cultura organizacional se basa en las interacciones de los diferentes grupos sociales en la organización, interacciones en las que se transmiten códigos culturales hasta conformar una "simbiosis entre las pautas marcadas por la organización, las propias creencias y los valores del grupo" (Capriotti, 2009, 24). Por ello se establece lo que Capriotti llama una estructura suprapersonal, es decir, una estructura conformada por los códigos culturales, producto de la simbiosis, que no pueden ser modificados fácilmente por las personas cuando actúan de manera aislada, y que condicionan positiva o negativamente las acciones de los colaboradores porque actúan como filtros. Por lo tanto, la óptima gestión del conocimiento depende de la configuración de la cultura organizacional, porque esta puede posibilitar, inhibir o anular los procesos a partir de los valores que permean a las personas. Según Pérez-Montoro (2008, 141), hay tres factores que podrían ser inhibidores de la gestión del conocimiento: "la historia (o memoria) organizacional, la existencia de procedimientos estandarizados y los paradigmas organizacionales". Lo podrían ser dependiendo de la coherencia que haya entre las creencias y los valores (donde están anclados los paradigmas organizacionales) con aquellos paradigmas básicos para la gestión del conocimiento.

Por ello, para que se dé la gestión del conocimiento, es necesaria una cultura organizacional flexible, dispuesta al cambio o al menos abierta a nuevas realidades, en la que los paradigmas organizacionales sean un filtro que canalice las iniciativas hacia los contextos propios de cada realidad organizacional, pero que incentive esos cambios que posibilitan el desarrollo estratégico de la organización.

Eso implica alinear la cultura con el propósito de la preparación para nuevas realidades en las que se compense los procedimientos estructurados con paradigmas de flexibilidad. También implica modificar estándares arraigados en los colaboradores por la costumbre, los hábitos y las tradiciones mediante nuevos paradigmas que se consolidan en principios, valores e ideas. Para esto, la comunicación es un elemento catalizador importante. 
Procesos de comunicación interna como alimentadores de la interacción social abierta al conocimiento

Si bien la comunicación ha ido ganando relevancia al ser un proceso transversal en las organizaciones, muchas veces ha sido comprendida meramente como un mediador funcionalista, es decir, como garante de la realimentación de los flujos de información dentro de los procesos. Sin embargo, como dice Fonseca $(2000,50)$, "el papel de la comunicación pasa a ser fundamental para comprender el comportamiento y la cultura organizacional, con vistas a aumentar la fuerza competitiva de la empresa en un escenario de constantes cambios". Este papel surge justamente de la capacidad de influir directamente la cultura en las interacciones sociales, las conductas y las formas de asumir los principios organizacionales. La comunicación pasa a generar climas de confianza en procesos de comunicación que logren la comprensión mutua de personas, procesos o entornos, sensibilización sobre nuevas formas de ver el mundo y el diálogo necesario para aprovechar las diferencias en torno a las metas organizacionales. Según Chillida (2007), estos climas de confianza en donde hay sinergia de esfuerzos y voluntades dan cierta libertad para actuar, evitando la saturación de consultas con las instancias y permitiendo que los diálogos sean más productivos. Esto lleva a la conformación de una autonomía basada en la confianza que los directivos tienen en sus colaboradores y que ellos tienen en sí mismos, tanto para el empoderamiento como para la participación y el aprendizaje continuo.

El conocimiento, entendido como un activo y como un proceso (Pérez-Montoro, 2008), puede ser gestionado dependiendo de las dinámicas relacionales de la organización porque:

La comunicación al interior de las empresas puede acelerar, detener o anular los procesos de innovación y de generación de conocimiento, ya que de la estructura de la comunicación organizacional dependen el: 1. Entendimiento de las políticas y objetivos de la empresa. 2. Establecimiento de rutinas eficientes de trabajo. 3 . Sistema de documentación de la empresa. 4. Flujo dinámico de información entre los niveles de la empresa 5. Mejoramiento de la curva de aprendizaje (Fontalvo, Quejada, \& Puello, 2011, 157).

Sin embargo, la comunicación busca establecer relaciones que van más allá de los flujos de información y que buscan responder al modelo de comunicación simétrico bidireccional de Grunig y Hunt (2003). Esto conduce a una toma de decisiones que "corresponde más a una búsqueda de acuerdos, que a la distribución de poderes" (Preciado, 2007, 39). Según este modelo, la comunicación directiva, es decir, entre líderes o directivos con colaboradores, juega un papel fundamental en el desarrollo de actitudes básicas, especialmente en la participación y el empoderamiento (Preciado, 2007), que redundan en la comunicación interpersonal en la que se promueve la libertad para hablar y compartir experiencias, dar opiniones e incluso ser propositivos.

Con ello, la comunicación se constituye en un facilitador del proceso educativo tanto en la formación y capacitación de los colaboradores como en las actitudes necesarias para que, desde el aprendizaje continuo, se influya en la cultura organizacional para la conformación de un ambiente propicio para la gestión del conocimiento.

\section{Conclusiones}

En la construcción de modelos propios para la gestión del conocimiento, las organizaciones deben dar relevancia al componente social, considerando los valores y actitudes de sus colaboradores, así como los procesos comunicacionales que fundamentan la cultura organizacional.

La comunicación interna permite las interacciones sociales necesarias para la transformación del conocimiento; dicha comunicación debe ser bidireccional y propiciar actitudes como apertura, confianza, disposición al conocimiento y empowerment.

Es necesario que en el diseño de la estrategia comunicacional y en el relacionamiento de 
los directivos con sus empleados, se genere y fomente la apropiación de espacios de participación y empoderamiento, que sirvan para fortalecer los valores necesarios para la gestión del conocimiento.

Para activar la motivación y el compromiso por parte de las personas -centro de la gestión del conocimiento- es necesario que se generen procesos de aprendizaje con sentido, en los cuales se vinculen los diferentes componentes de la cultura organizacional y de los valores que prevalecen en la misma.

\section{Referencias bibliográficas}

- Arboníes, Á. (2006). Conocimiento para innovar: cómo evitar la miopía en la gestión del conocimiento. Madrid: Díaz de Santos.

- Awad, E. M. \& Ghaziri, H. M. (2004). Knoledge Management. New Jersey: Pearson Education.

- Capriotti, P. (2009). Branding Corporativo. Fundamentos para la gestión estratégica de la identidad corporativa. Santiago de Chile: Colección de libros de la empresa.

- Chillida, G. (2007). La comunicación organizacional: Clave para la gestión del conocimiento eficiente. Razón y Palabra(55). Obtenido de http://www.razonypalabra.org.mx/ anteriores/n55/gchillida.html

- Fonseca, R. (2000). Gestión de calidad y del conocimiento: la comunicación. Revista Prisma (13), 43-51.

- Fontalvo, T.; Quejada, R. \& Puello, J. (2011). La comunicación organizacional como agente dinamizador del mejora continua en los sistema de gestión. Universidad Autónoma del Caribe. Encuentros., 09(2), 147-160.

- García-Morales, V. J.; Matías-Reche, F. \& Verdú-Jover, A. J. (2011). Influence of Internal Communication on Technological Proactivity, Organizational Learning, and Organizational Innovation in the Pharmaceutical sector. International Communication Association. Journal of Communication, 150-177.

- Grunig, J. E. \& Hunt, T. (2003). Dirección de relaciones públicas. Barcelona: Gestión 2000.

- Lopez, M.; Marulanda, C. \& Isaza, G. (mayoagosto de 2011). Cultura organizacional y gestión del cambio y de conocimiento en organizaciones de Caldas. Revista Virtual Universidad Católica del Norte, (33), 117-139.

- Nonaka, I. \& Takeuchi, H. (1999). La organización creadora de conocimiento. México: Oxford University Press.

- Pérez-Montoro, M. (2008). Gestión del conocimiento en las organizaciones: Fundamentos, metodología y praxis. España: Trea.

- Preciado, Á. (2007). Comunicación directiva. Influencia del estilo de dirección en la comunicación interna de las organizaciones. Medellín: Universidad Pontificia Bolivariana.

- Quintanilla Pardo, I. (2003). Empresas y personas. Madrid: Ediciones Díaz de Santos.

- Schein, E. H. (1988). La cultura empresarial y el liderazgo. Barcelona: Plaza \& Janés Editores.

- Valhondo, D. (2010). Gestión del conocimiento: del mito a la realidad. Madrid: Díez de Santos. 